\title{
Modelagem matemática para sistema de tratamento de esgoto doméstico com remoção simultânea de matéria orgânica e nitrogênio
}

\author{
Mathematical modeling for domestic sewage treatment system \\ with simultaneous removal of organic matter and nitrogen
}

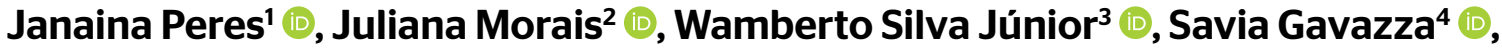 Lourdinha Florencio ${ }^{4} \oplus$, Mario Kato ${ }^{4 *}($ ()

\section{RESUMO}

Neste trabalho, avaliou-se o uso do modelo matemático ASM3 para um sistema piloto de reator compartimentado anaeróbio/anóxico e aeróbio tipo MBBR, utilizado para a remoção simultânea de matéria orgânica e nitrogênio em esgoto doméstico. A simulação computacional foi implementada pelo software ASIM. Selecionou-se um conjunto padrão de parâmetros para o modelo, com uma combinação dos valores calibrados e dados da literatura. Com a modelagem computacional foi possível incluir as características operacionais próprias do sistema de tratamento e, embora o modelo não tenha tido sua capacidade de previsão dos parâmetros validada, foi possível verificar a aplicabilidade do ASM3 para as fases operacionais definidas neste estudo.

Palavras-chave: modelo ASM3; software ASIM; reator compartimentado anaeróbio/anóxico e aeróbio MBBR.

\begin{abstract}
In this work, the use of the ASM3 mathematical model for a pilot system of anaerobic/anoxic and aerobic MBBR compartmentalized reactor, for the simultaneous removal of organic matter and nitrogen in domestic sewage, was evaluated. The simulation was implemented by the ASIM software. A standard set of parameters was selected for the model, with a combination of calibrated values and literature data. With the computational modeling, it was possible to include the operational characteristics of the treatment system and, although the model capacity to predict the parameters could not be validated. Nevertheless, it was possible to verify the applicability of the ASM3 model for the operational phases defined in this study.
\end{abstract}

Keywords: ASM3 model; ASIM software; anaerobic/anoxic and aerobic MBBR compartmentalized reactor.

\section{INTRODUÇÃO}

Padrões nacionais e internacionais de qualidade da água têm se tornado cada vez mais rígidos, o que exige o desenvolvimento de tecnologias de tratamento mais eficientes com o mínimo de impacto ambiental e o menor custo possível. Uma alternativa para o uso de tecnologias convencionais é a implantação de reatores compartimentados ou integrados que combinam processos anaeróbios, anóxicos e aeróbios, na tentativa de maximizar as vantagens que cada sistema possui e, ao mesmo tempo, minimizar seus pontos negativos (CHAN et al., 2009).

Com o objetivo de alcançar melhor estabilidade e maior eficiência no processo, já foram propostas diversas combinações de tratamento anaeróbio e aeróbio. O grande desafio da aplicação do tratamento com sistemas combinados é encontrar a condição ótima para cada um. Segundo Ferrai, Guglielmi e Andreottola (2010), os critérios de projeto utilizados para esses reatores ainda são bastante empíricos, confiando-se principalmente em parâmetros como a carga superficial aplicada (carga de poluentes por unidade de superfície dos suportes) e o tempo de detenção hidráulica, para alcançar a qualidade desejada do efluente.

A modelagem matemática de reatores compartimentados é uma ferramenta importante para previsões de desempenho ou comparações de alternativas de tratamento, propiciando o controle e a otimização de tecnologias. Outra vantagem dos modelos matemáticos refere-se à potencial economia na construção e nos custos de operação e manutenção (CHRISTOFOLETTI, 2004).

'Universidade Federal Rural de Pernambuco - Recife (PE), Brasil.

${ }^{2}$ Instituto Federal de Educação, Ciência e Tecnologia de Pernambuco - Recife (PE), Brasil.

3!nstituto Federal de Educação, Ciência e Tecnologia da Paraiba - Monteiro (PB), Brasil.

${ }^{4}$ Universidade Federal de Pernambuco - Recife (PE), Brasil.

*Autor correspondente: mariotk_kato@yahoo.com.br, mario.kato@ufpe.br

Conflitos de interesse: os autores declaram não haver conflito de interesses.

Financiamento: Conselho Nacional de Desenvolvimento Científico e Tecnológico (CNPq) e Fundação de Amparo à Ciência e Tecnologia de Pernambuco (FACEPE) (projeto

PRONEX/NUTREL), Coordenação de Aperfeiçoamento de Pessoal de Nível Superior (CAPES) (bolsas) e Financiadora de Estudos e Projetos (FINEP) (projeto RENTED).

Recebido: 21/06/2018 - Aceito: 02/09/2O2O - Reg. ABES: 20180025 
Entre os diversos modelos matemáticos desenvolvidos, os mais utilizados e com grande difusão no campo científico e prático de tratamento de águas residuárias são os Activated Sludge Models (ASM) desenvolvidos pela International Water Association (IWA). Entre eles, o ASM3, composto de 12 processos e 13 componentes ou variáveis, tem sido muito utilizado na modelagem do tratamento de esgoto em razão de sua facilidade de aplicação, calibração e por corrigir carências contidas no modelo ASM1 (GERNAEY et al., 2004).

Dessa forma, o principal objetivo deste estudo foi implementar o modelo ASM3 por meio dos dados de monitoramento de um reator experimental compartimentado anaeróbio/anóxico e aeróbio, em escala piloto, para remoção simultânea de matéria orgânica e nitrogênio em esgoto doméstico, com o intuito de verificar sua aplicabilidade, bem como avaliar os aspectos positivos e negativos da implantação do modelo ASM3 no reator em estudo.

\section{METODOLOGIA}

O reator experimental compartimentado (anaeróbio/anóxico e aeróbio) (Figura 1) utilizado para o estudo foi operado na área experimental da estação de tratamento de esgotos (ETE) da Mangueira, localizada em Recife, Pernambuco. Os dados de operação e monitoramento foram obtidos de um dos reatores utilizados em experimento prévio em escala piloto, desenvolvido por Morais (2015).

$\mathrm{O}$ reator avaliado, denominado $\mathrm{RC} 1$, era vertical, construído em tubo PVC tipo comercial, com diâmetro de 0,40 m, altura útil de 3,10 m e volume total de $390 \mathrm{~L}$. O leito era dividido em dois compartimentos separados por placas de aço inox perfuradas. O primeiro compartimento era anaeróbio/anóxico, com altura de 0,70 m e volume de $90 \mathrm{~L}$, para a remoção de matéria orgânica. $\mathrm{O}$ segundo compartimento, aeróbio, operou como reator MBBR (moving bed biofilm reactor), media $2,20 \mathrm{~m}$ de altura e tinha volume de $300 \mathrm{~L}$. O compartimento aeróbio recebeu meio suporte móvel feito de polietileno com densidade de $0,95 \mathrm{~g} . \mathrm{cm}^{-3}$, com forma cilíndrica de $25 \mathrm{~mm}$ de diâmetro e $12 \mathrm{~mm}$ de altura, e razão de recheio de 0,55 com a finalidade de promover a nitrificação. Para a aeração do compartimento aeróbio foi utilizado o difusor de ar de bolha fina HD Ф $270 \mathrm{~mm}$. O efluente nitrificado foi recirculado com taxa de 1,5 para o compartimento anóxico, a fim de promover a desnitrificação, utilizando o

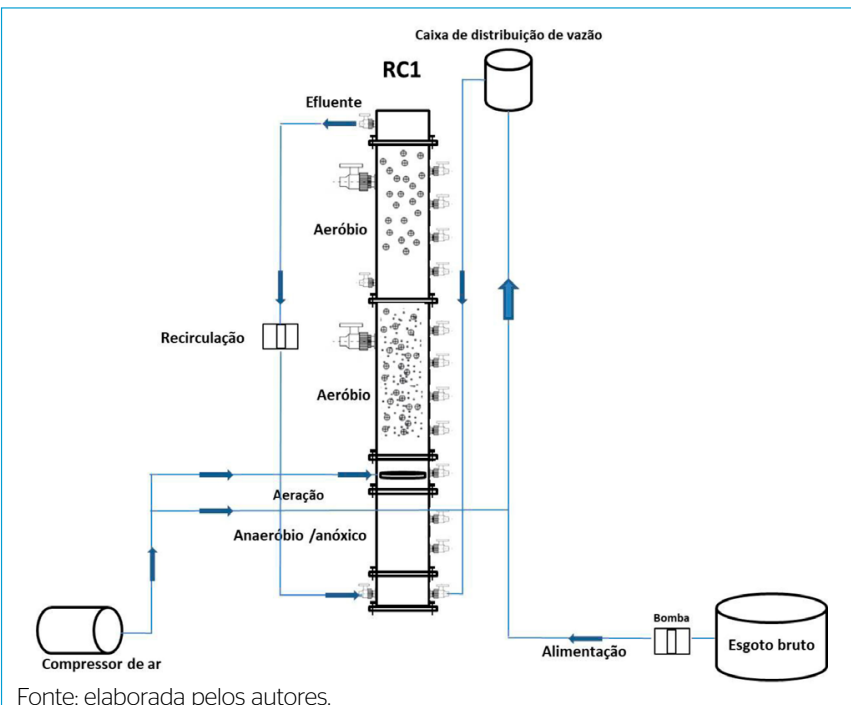

Figura 1 - Esquema do reator compartimentado RC1, anaeróbio/anóxico e aeróbio tipo MBBR. próprio esgoto bruto como doador de elétrons. O reator RC1 foi alimentado com esgoto doméstico após tratamento preliminar.

Os dados para a modelagem matemática foram obtidos da melhor fase operacional do reator compartimentado, em que foi aplicado um tempo de detenção hidráulica de $12 \mathrm{~h}$, taxa de recirculação de 1,5 e velocidade ascensional de $0,64 \mathrm{~m} \cdot \mathrm{h}^{-1}$. O oxigênio dissolvido no compartimento aeróbio foi de 2,5 mg.L $\mathrm{L}^{-1}$. Nessa fase, o reator compartimentado RC1 foi operado por 153 dias (Morais, 2015).

A Tabela 1 apresenta a matriz estequiométrica $v_{\mathrm{j}, \mathrm{i}}$ do modelo ASM3 em conjunto com a matriz de composição $i_{\mathrm{k}, \mathrm{i}}$ como proposto por Gujer e Larsen (1995). Essa matriz de composição pode ser lida da seguinte forma: $i_{2,3}$ preenchida com o símbolo $i_{\mathrm{N}, \mathrm{SS}}$ indica que qualquer grama de demanda química de oxigênio (DQO) na forma de substrato prontamente biodegradável $\left(\mathrm{S}_{\mathrm{S}}\right)$ contém $i_{\mathrm{N}, s \mathrm{~S}}$ gramas de nitrogênio. $\mathrm{O}$ índice $k=2 \mathrm{diz}$ respeito ao segundo conservativo, o nitrogênio. $\mathrm{O}$ índice $i=3 \mathrm{diz}$ respeito ao terceiro componente, o $\mathrm{S}_{\mathrm{S}}$. Este é medido em termos de g DQO e o conservativo nitrogênio é expresso em g $\mathrm{N}$. Dessa forma, $i_{\mathrm{N}, \mathrm{SS}}$ indica a composição de $\mathrm{S}_{\mathrm{S}}$ em relação ao nitrogênio, daí $i_{\mathrm{k}, i}$ ser chamada de matriz de composição.

Todos os valores de $x_{\mathrm{j}}, y_{\mathrm{j}} \mathrm{e} z_{\mathrm{j}}$ podem ser obtidos a partir da equação de conservação (Equação 1) para os três conservadores $k$ : demanda teórica de oxigênio (ThOD), nitrogênio e carga iônica.

$\sum_{i} v j, i \cdot i k, i=0 \quad$ para $i=1$ a 12

$\mathrm{O}$ coeficiente estequiométrico para nitrogênio gasoso $\left(\mathrm{S}_{\mathrm{N} 2}\right)$ em qualquer processo de desnitrificação é o valor negativo do coeficiente para nitrogênio na forma de nitrato + nitrito $\left(\mathrm{S}_{\mathrm{NoX}}\right)$. Os coeficientes de composição de ThOD para $\mathrm{S}_{\mathrm{N} 2}\left(-1,71 \mathrm{~g}\right.$ de THOD $\left.\left(\mathrm{g} \mathrm{N}_{2}\right)^{-1}\right)$ e para $\mathrm{S}_{\mathrm{Nox}}\left(-4,57 \mathrm{~g}\right.$ de ThOD $\left.\left(\mathrm{g} \mathrm{NO}_{3}-\mathrm{N}\right)^{-1}\right)$, bem como para oxigênio dissolvido $-\mathrm{S}_{\mathrm{O} 2}\left(-1 \mathrm{~g}\right.$, ThOD $\left.\left(\mathrm{g} \mathrm{O}_{2}\right)^{-1}\right)$, são negativos para doadores de elétrons em razão do potencial redox de ThOD.

Os coeficientes estequiométricos para sólidos suspensos totais $\left(\mathrm{X}_{\mathrm{SST}}\right)$ foram obtidos pela Equação 2.

$\sum_{i} v j, i . i 4, i=v j, 13$ para $i=8$ a 12

A produção de energia bioquímica (ATP) na respiração anóxica é menor do que na respiração aeróbia. Isso conduz ao fato de que os coeficientes de rendimento aeróbio ( $Y_{\mathrm{STO} O \mathrm{O} 2}$ e $\left.Y_{\mathrm{H}, \mathrm{O} 2}\right)$ excedem os coeficientes de rendimento anóxicos $\left(Y_{\mathrm{STONOX}}\right.$ e $\left.Y_{\mathrm{H}, \mathrm{NOX}}\right)$. Assim, para o rendimento anóxico de energia, com $\eta_{\text {anóxico }}=0,70$ do rendimento energético aeróbio, a seguinte relação energética (Equação 3) pode ser aplicada:

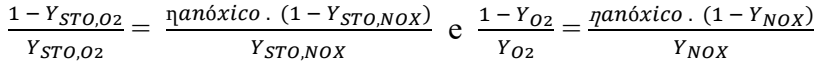

As expressões cinéticas do modelo ASM3 foram baseadas em funções de comutação (termos hiperbólicos ou de saturação, equações de Monod, $\mathrm{S} /(\mathrm{Ks}+\mathrm{S})$, sendo $\mathrm{S}$ a concentração do substrato limitante e Ks a constante de saturação) para todos os compostos solúveis consumidos. Essa forma de expressão cinética foi escolhida não por causa de evidência experimental, $\mathrm{e}$ sim por conveniência matemática: essas funções de comutação interrompem toda a atividade biológica, como guias, quando as concentrações do processo se aproximam de zero, o que caracteriza uma importante diferença entre 
os modelos ASM1 e ASM3. A inibição foi modelada com $1-\mathrm{S} /(\mathrm{Ks}+\mathrm{S})=$ Ks / $(\mathrm{Ks}+\mathrm{S})$. A Tabela 2 apresenta um resumo das expressões cinéticas do modelo ASM3.

Diversos autores já utilizaram, calibraram e/ou adaptaram o modelo ASM3 e suas equações para inúmeras configurações de reatores compartimentados. Brockmann et al. (2013) desenvolveram um modelo para reatores MBBR, para os quais foram modelados os processos de conversão bioquímica utilizando algumas variáveis e processos do modelo ASM3. A análise após simulação computacional do modelo mostrou que uma boa caracterização de águas residuárias em relação ao substrato orgânico é importante para modelar reatores de biofilme nitrificantes; assim, podem representar adequadamente as concentrações de sólidos no efluente e na biomassa. No entanto, para a calibração dinâmica, abordou-se que a evolução da biomassa do biofilme no tempo ainda não pôde ser descrita como exatamente acontece, o que revelou ainda grandes dificuldades na modelagem de reatores com biofilme.

Já Ferrai, Guglielmi e Andreottola (2010) utilizaram uma versão adaptada e estendida do modelo ASM3 (SIN et al., 2005), que previa a difusão do transporte de massa, para modelar um reator MBBR compartimentado anóxico e aeróbio tratando esgoto doméstico, obtendo-se bons resultados após os ajustes de calibração.

\section{Obtenção dos valores das variáveis para uso do modelo ASM3}

O modelo ASM3 utiliza 13 variáveis e os valores para a sua aplicação neste trabalho foram obtidos por meio de análises laboratoriais, na literatura ou por balanço de massa. A Tabela 3 discrimina a origem da obtenção daqueles valores.

Para a obtenção dos valores para $\mathrm{S}_{\mathrm{N} 2}$, foi realizado o balanço de massa dos compostos nitrogenados presentes no afluente. Para a determinação dos valores de cada fração da DQO, da biomassa e do produto de armazenamento celular, foram utilizados como referência aqueles citados por Koch et al. (2000). A Tabela 4 especifica os valores de referência das frações supracitadas.

\section{Simulação computacional e estatística}

O software utilizado para as simulações computacionais foi o ASIM (Activated Sludge SIMulation Program) (ASIM, 2016). O modelo ASIM apresenta capacidade de simular unidades de estações de tratamento de esgoto com processo de nitrificação. Além disso, no banco de dados desse modelo existem vários tipos de reatores, o que permite a montagem de unidades muito próximas a um reator em escala real.

As simulações computacionais no modelo ASM3 foram realizadas em duas etapas. Na primeira etapa foram utilizados os valores cinéticos e estequiométricos propostos por Henze et al. (2000) e na segunda etapa foram aplicados os

Tabela 1 - Matriz estequiométrica do modelo ASM3.

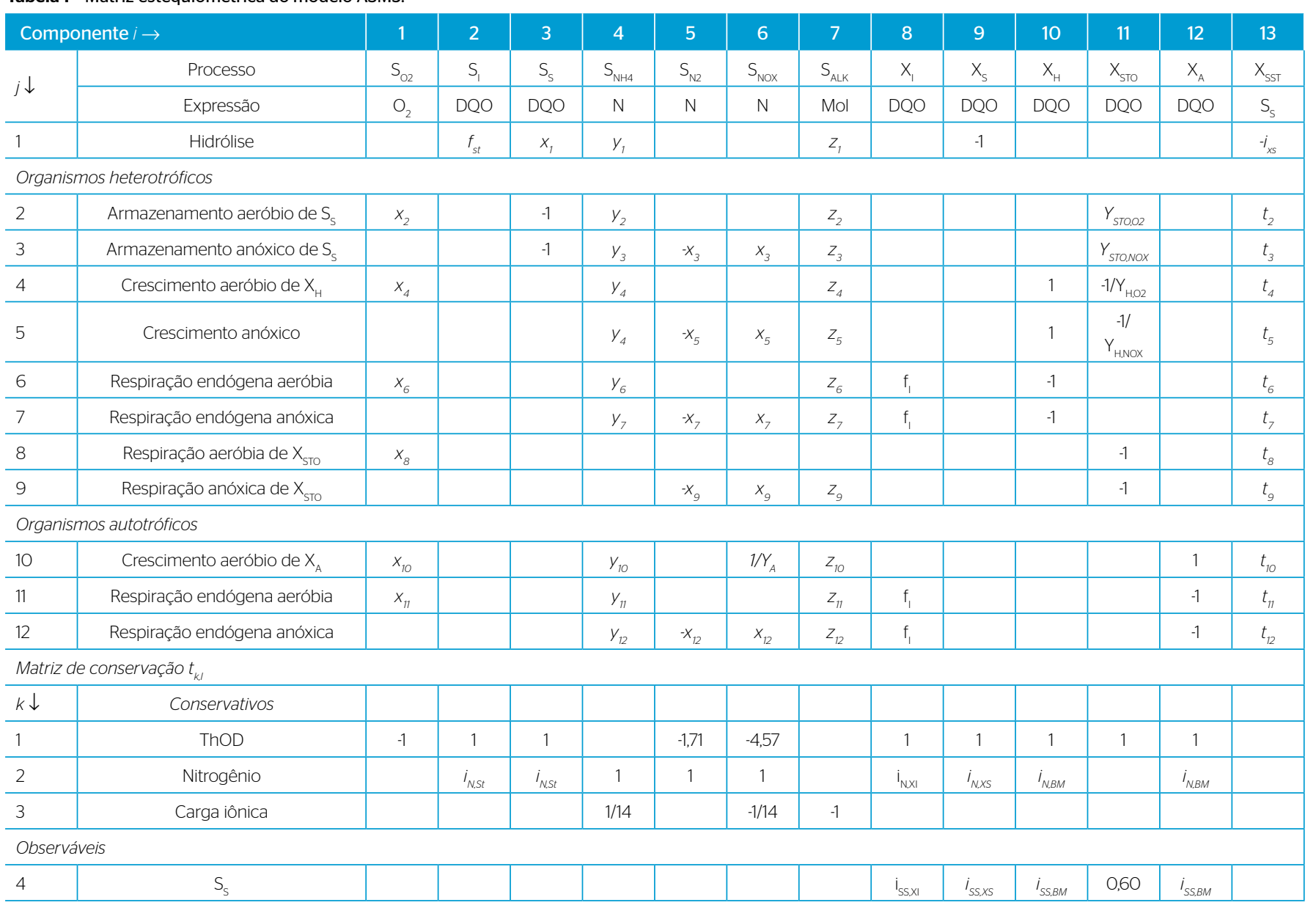

$\mathrm{S}_{\mathrm{O} 2}$ : oxigênio dissolvido; $\mathrm{S}$ : material orgânico solúvel inerte; $\mathrm{S}_{\mathrm{S}}$ : substrato prontamente biodegradável; $\mathrm{S}_{\mathrm{NH}}$ : :itrogênio na forma $\mathrm{NH}_{4}+$ e $\mathrm{NH}_{3}$; $\mathrm{S}_{\mathrm{N} 2}$ : nitrogênio gasoso; $\mathrm{S}_{\mathrm{Nox}}$ : nitrogênio na forma de nitrato + nitrito; $S_{A L K}$ : alcalinidade; $X_{i}$ material orgânico particulado inerte; $X_{S}$ : substrato lentamente biodegradável; $X_{H}$ : biomassa heterotrófica; $X_{\text {STo: }}$ : produto de armazenamento celular interno; $\mathrm{X}_{\mathrm{A}}$ : biomassa autotrófica; $\mathrm{X}_{\mathrm{SST}}$ sólidos suspensos totais; $\mathrm{DQO}$ : demanda química de oxigênio; ThOD: demanda teórica de oxigênio. Fonte: Henze et al. (2000). 


\section{RESULTADOS E DISCUSSÃO}

Com os gráficos gerados e a aplicação do teste estatístico $t$ de Student (SOKAL; ROHLF, 1970; ISAZADEH et al., 2015; SILVA; SILVA; SARTI, 2017), foi observado que as curvas simuladas e reais não foram estatisticamente iguais para nenhum dos parâmetros, tanto com o uso do modelo ASM3 original quanto com o ASM3 de Koch et al. (2000).

\section{Demanda química de oxigênio total}

A DQO total é uma medida extremamente importante, uma vez que é o parâmetro utilizado para representar a matéria orgânica no sistema. A fase de operação estudada deste reator foi a que apresentou a melhor eficiência de remoção, tanto para matéria carbonácea (eficiência média de remoção DQO bruta/bruta e DQO bruta/filtrada, afluente/efluente, de $52 \pm 15 \%$ e 81 $\pm 7 \%$, respectivamente) quanto para a matéria nitrogenada (60\%). Os valores médios no afluente de DQO bruta e de N-NTK foram de $475 \pm 174 \mathrm{mg} . \mathrm{L}^{-1} \mathrm{e}$ $40 \pm 7$ mg. $\mathrm{L}^{-1}$, respectivamente. As grandes oscilações observadas nas concentrações de DQO no efluente (Figura 2) podem ser atribuídas às próprias oscilações no afluente, não ao desempenho do reator em si, o qual mostrou boa eficiência de remoção.

Ao analisar a Figura 2, observou-se que as concentrações medidas no efluente ficaram abaixo da curva das concentrações simuladas, tanto pelo modelo ASM3 original quanto pelo ASM3 de Koch. Ao aplicar o teste estatístico $t$ de Student, ao nível de 95\% de probabilidade, rejeitou-se a hipótese de que as simulações propostas, tanto pelo ASM3 original quanto pelo de Koch et al. (2000), fossem adequadas à situação real; para esse parâmetro, foram obtidos valores de $t=-8,586$ e $t=-6,28$, respectivamente. $\mathrm{O}$ valor tabelado de $t$ para 15 graus de liberdade com significância igual a 0,05 é de $\pm 1,753$.

Tabela 3 - Método de obtenção dos valores das variáveis utilizadas no modelo ASM3.

\begin{tabular}{l|c|c} 
Sigla & Descrição & Obtenção \\
\hline $\mathrm{S}_{\mathrm{O} 2}$ & Oxigênio dissolvido & Análise do monitoramento \\
\hline $\mathrm{S}_{1}$ & Material orgânico solúvel inerte & Koch et al. (2000) \\
\hline $\mathrm{X}_{1}$ & Material orgânico particulado inerte & Koch et al. (2000) \\
\hline $\mathrm{S}_{\mathrm{S}}$ & Substrato prontamente biodegradável & Koch et al. (2000) \\
\hline $\mathrm{X}_{\mathrm{S}}$ & Substrato lentamente biodegradável & Koch et al. (2000) \\
\hline $\mathrm{X}_{\mathrm{H}}$ & Biomassa heterotrófica & Koch et al. (2000) \\
\hline $\mathrm{X}_{\mathrm{A}}$ & Biomassa autotrófica & Koch et al. (2000) \\
\hline $\mathrm{X}_{\mathrm{STO}}$ & Produto de armazenamento celular interno & Koch et al. (2000) \\
\hline $\mathrm{S}_{\mathrm{NOX}}$ & Nitrogênio na forma de nitrato + nitrito & Análise do monitoramento \\
\hline $\mathrm{S}_{\mathrm{NH4}}$ & Nitrogênio na forma NH${ }_{4}^{+}$e $\mathrm{NH}_{3}$ & Análise do monitoramento \\
\hline $\mathrm{S}_{\mathrm{N} 2}$ & Nitrogênio gasoso & Balanço de massa \\
\hline $\mathrm{X}_{\mathrm{SST}}$ & Sólidos suspensos totais & Análise do monitoramento \\
\hline $\mathrm{S}_{\mathrm{ALK}}$ & Alcalinidade & Análise do monitoramento \\
\hline
\end{tabular}

Fonte: elaborada pelos autores.
Pelo comportamento apresentado, foi notório que a matéria orgânica afluente é oxidada mais rapidamente do que o proposto pelo ASM3, tanto na condição original quanto com as constantes de Koch. Uma explicação para essa ocorrência, além da forte oscilação da DQO afluente anteriormente relatada, pode ser também a temperatura tropical em que o reator compartimentado operou, que torna a atividade de microrganismos mais intensa e aumenta a velocidade de oxidação da matéria orgânica, o que, consequentemente, diminui a DQO total no efluente.

$\mathrm{O}$ modelo foi proposto para atuar sem restrições de $8-23^{\circ} \mathrm{C}$ (o reator em questão operou com temperatura média de $29,3^{\circ} \mathrm{C}$ ) e mesmo com correções de coeficientes de temperatura realizadas pelo próprio simulador, não foi possível representar as condições reais.

\section{Nitrato + nitrito e nitrogênio amoniacal}

A Figura 3 mostra que as curvas das concentrações simuladas para $\mathrm{S}_{\mathrm{NOX}}$ divergem muito dos dados de monitoramento do efluente, tanto para a simulação com o modelo ASM3 original quanto para o ASM3 de Koch et al. (2000), tendo apresentado valores de $t=2,708$ e $t=2,545$, respectivamente. Para esse parâmetro, $o$ valor tabelado de $t$ para 15 graus de liberdade com significância igual a 0,05 é de $\pm 1,753$.

As curvas simuladas apresentaram concentrações geralmente próximas de zero, o que não condiz com o observado no sistema real. O modelo considera em sua estrutura a ocorrência da desnitrificação total, mas isso não foi observado no sistema real, uma vez que em alguns pontos se observaram concentrações relativamente altas de nitrato + nitrito no efluente final.

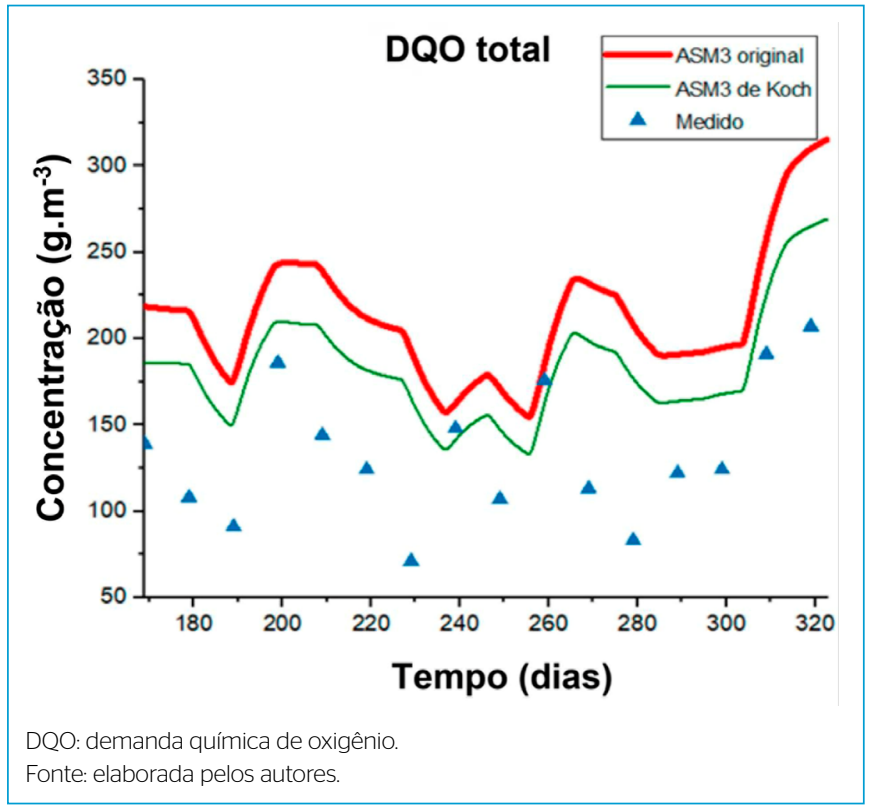

Figura 2 - Demanda química de oxigênio total efluente: valores medidos e os obtidos da simulação com o ASM3 original e de Koch et al. (2000).

Tabela 4 -Valores de referência das frações da demanda química de oxigênio total para o modelo ASM3.

\begin{tabular}{l|c|c|c|c|c|c|c|c|}
\hline Fração & $\mathrm{S}_{\mathrm{I}}$ & $\mathrm{S}_{\mathrm{S}}$ & $\mathrm{X}_{\mathrm{I}}$ & $\mathrm{X}_{\mathrm{S}}$ & $\mathrm{X}_{\mathrm{H}}$ & $\mathrm{X}_{\mathrm{A}}$ & $\mathrm{X}_{\mathrm{sto}}$ \\
\hline$\%$ & 6 & 10 & 20 & 55 & 9 & 0 \\
\hline
\end{tabular}

$\mathrm{S}_{\mathrm{i}}$ : material orgânico solúvel inerte; $\mathrm{S}_{\mathrm{S}}$ : substrato prontamente biodegradável; $\mathrm{X}_{\mathrm{i}}$ : material orgânico particulado inerte; $\mathrm{X}_{\mathrm{S}}$ : substrato lentamente biodegradável; $\mathrm{X}_{\mathrm{H}}$ : biomassa heterotrófica; $X_{\mathrm{A}}$ : biomassa autotrófica; $\mathrm{X}_{\text {STO }}$ : produto de armazenamento celular interno.

Fonte: Koch et al. (2000). 
Já a Figura 4 apresenta as simulações e os dados de monitoramento para a variável "nitrogênio amoniacal". Observou-se que a maioria dos pontos analisados estava abaixo das curvas simuladas com os modelos ASM3 original e ASM3 de Koch. Ao aplicar o teste $t$, ao nível de $95 \%$ de probabilidade, rejeitou-se a hipótese de que as simulações propostas fossem adequadas à situação real, tendo apresentado o valor calculado de $t=-3,803$ para a simulação com ASM3 original e de $t=$ -2,808 para a simulação com o modelo ASM3 de Koch et al. (2000). O valor tabelado de $t$ para 15 graus de liberdade com significância igual a 0,05 é de $\pm 1,753$.

\section{Alcalinidade total}

O gráfico da variável “alcalinidade total” (Figura 5) mostrou que as curvas das concentrações simuladas, tanto pelo modelo ASM3 original quanto pelo ASM3

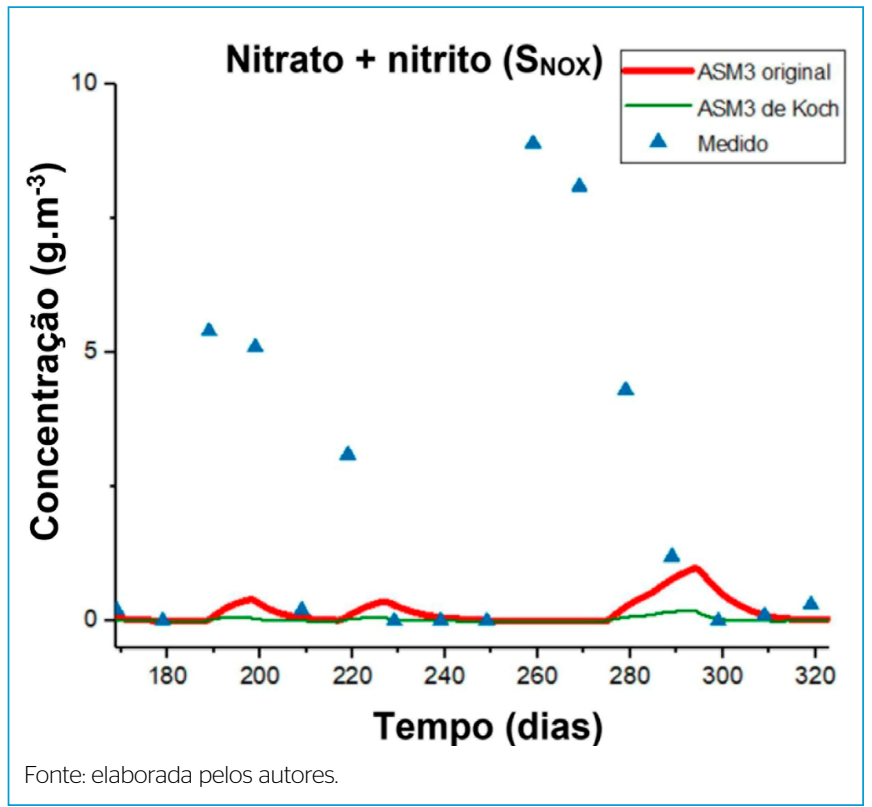

Figura 3 - Nitrogênio na forma de nitrito e nitrato efluente simulado com os modelos ASM3 original e de Koch et al. (2000) e valores medidos.

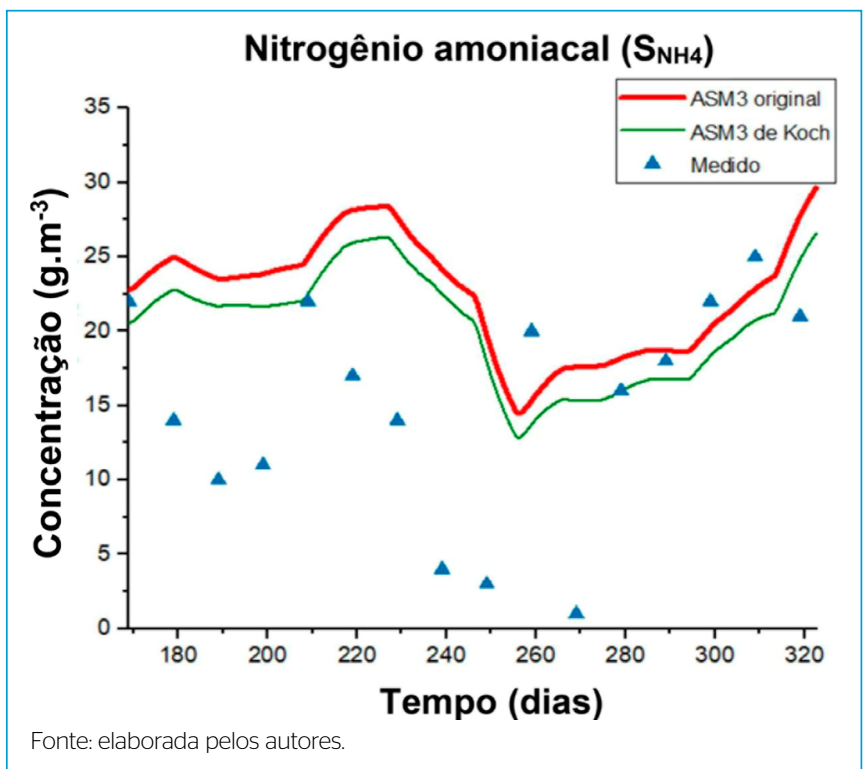

Figura 4 - Nitrogênio amoniacal efluente simulado com os modelos ASM3 original e de Koch et al. (2000) e valores medidos. de Koch, estão próximas aos pontos de monitoramento do efluente final do reator, embora esses apresentem alguns pontos divergentes.

O consumo de alcalinidade é proveniente, principalmente, da nitrificação. Logo, nas situações em que os pontos de monitoramento apresentaram valores abaixo da curva esperada, é entendido que o sistema real nitrificou, mas não realizou a desnitrificação na proporção adequada para a estabilidade da alcalinidade, o que deixa clara uma desarmonia na cinética do modelo com a cinética real do reator.

Aplicando-se o teste $t$ a 95\% de probabilidade, a hipótese de que a simulação proposta pelo modelo para a variável “alcalinidade” fosse adequada à situação real foi rejeitada para os modelos ASM3 original e de Koch, com valores de $t$ calculado de $-2,481$ e $-1,777$, respectivamente. O valor tabelado de $t$ para 15 graus de liberdade com significância igual a 0,05 é de $\pm 1,753$.

\section{Sólidos suspensos totais}

Com a análise dos gráficos de $\mathrm{X}_{\mathrm{SST}}$ (Figura 6), observou-se que os pontos analisados do efluente real apresentaram concentrações abaixo das curvas das concentrações simuladas, o que indicaria desempenho melhor do que o esperado pelo modelo no caso da concentração de sólidos. Isso pode ter acontecido em razão de uma alta retenção de sólidos no interior do reator, justificada pela falta de inoculação inicial. A divergência entre os pontos medidos e as curvas simuladas, corroborada pelo teste $t$, revelou que os modelos ASM3 original e ASM3 de Koch também não foram representativos para esse parâmetro.

Portanto, o modelo ASM3 com seus parâmetros cinéticos padrão, bem como com os parâmetros cinéticos propostos por Koch e colaboradores, não puderam ser validados para o reator compartimentado anaeróbio/anóxico e aeróbio em estudo. A simulação de nenhuma das variáveis correspondeu aos dados de monitoramento. Sendo assim, foi necessário realizar ajustes de calibração de alguns parâmetros, a partir dos parâmetros cinéticos do ASM3 original e dos parâmetros propostos por Koch et al. (2000), na tentativa de validar o modelo ASM3 para o reator em questão.

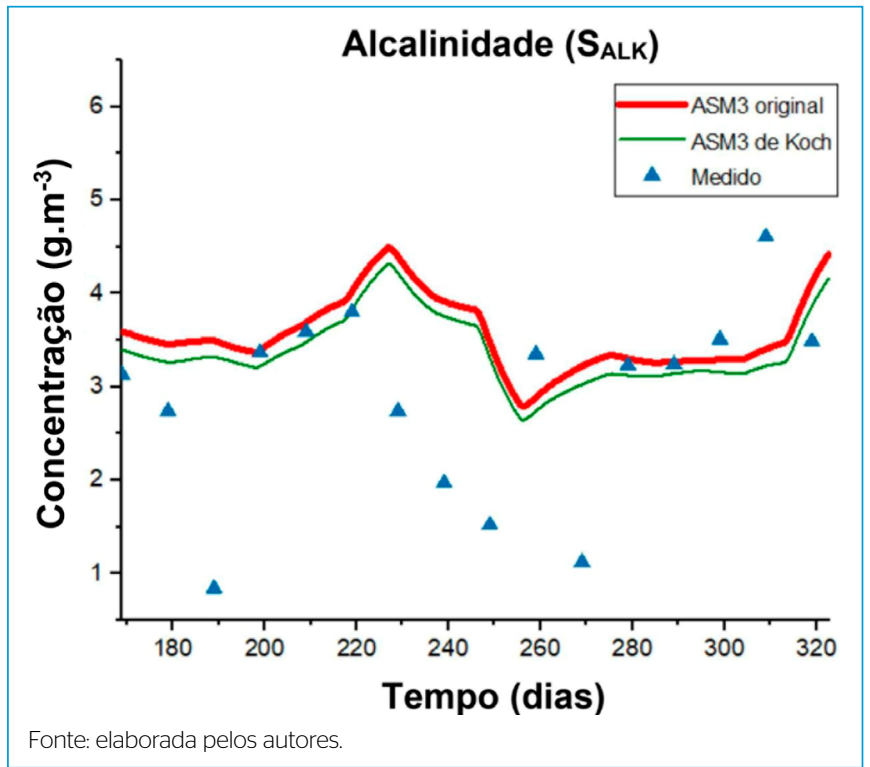

Figura 5 - Alcalinidade efluente simulada com os modelos ASM3 original e de Koch et al. (2000) e valores medidos. 


\section{Ajustes de calibração do modelo}

Para realizar os ajustes de calibração dos parâmetros cinéticos do modelo ASM3, inicialmente foram utilizados os valores padrão do ASM3 original.

As tentativas de calibração tiveram início pela alteração da taxa de crescimento heterotrófico anóxico $\left(\mu_{\text {Hanóx }}\right)$. Isso porque, segundo Henze et al. (2000), recomenda-se sempre iniciar as calibrações por aquelas taxas. Ainda, por ser o compartimento anaeróbio/anóxico o que tinha por função principal a remoção da matéria orgânica, iniciou-se então a calibração por essa taxa.

No ASM3 original, $\mu_{\text {Hanóx }}$ teve valor de 1,2 $\mathrm{d}^{-1}$. Como a temperatura média de operação do reator foi superior ao intervalo previsto pelo modelo, optou-se por se elevar consideravelmente o valor de $\mu_{\text {Hanóx }}$ para 2,0 d $\mathrm{d}^{-1}$, a fim de observar a forma como um aumento brusco impactaria nas simulações. No entanto, após simular dessa forma, não foi observado nenhum tipo de alteração relevante. Então, em seguida, optou-se por baixar drasticamente o valor de $\mu_{\text {Hanóx }}$ para $0,5 \mathrm{~d}^{-1}$, com o intuito de verificar se uma redução considerável traria modificações nas simulações. No entanto, também não se observou alterações significativas nas simulações, tanto para a DQO total quanto para as variáveis nitrogenadas.

Ficou evidente que a alteração do parâmetro $\mu_{\text {Hanóx }}$ não trouxe redução das concentrações nas curvas, para que fossem compatíveis estatisticamente com a curva da DQO total medida no efluente final do reator. Dessa forma, priorizou-se realizar alterações nos valores da taxa de crescimento heterotrófico aeróbio $\left(\mu_{H}\right)$ para observar as modificações que causariam nas curvas simuladas. É importante ressaltar que sempre se deve realizar uma iteração por vez, de forma a observar a influência de cada parâmetro sobre cada variável a ser calibrada.

O valor padrão de $\mu_{\mathrm{H}}$ no ASM3 original foi de 2,0 $\mathrm{d}^{-1}$. Dessa forma, a primeira tentativa de calibração foi elevar esse valor para $3,0 \mathrm{~d}^{-1}$. Observou-se, após as novas simulações, que a curva simulada para a DQO total ficou em um patamar mais baixo, mas ainda ficou longe de ser considerada validada. Em seguida, realizaram-se tentativas de aumentar o valor de $\mu_{\mathrm{H}}$ para 4,0, 4,5 e $5,0 \mathrm{~d}^{-1}$, tendo-se alcançado o melhor resultado com a simulação de $4,0 \mathrm{~d}^{-1}$; no entanto, ainda muito longe de se realizar a validação do modelo.

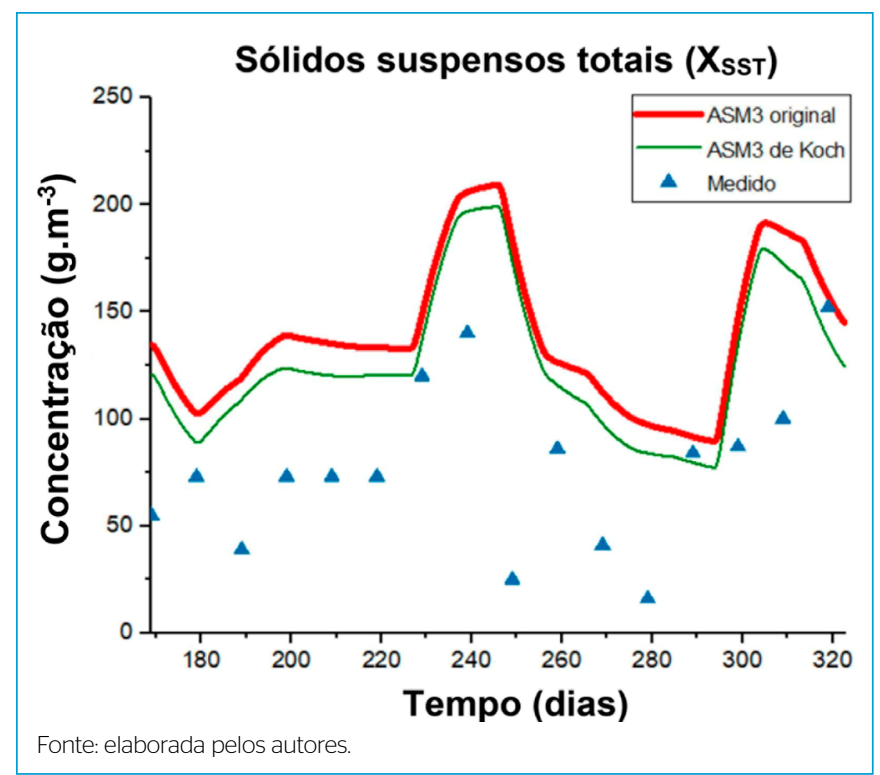

Figura 6 - Sólidos suspensos totais efluentes simulados com os modelos ASM3 original e de Koch et al. (2000) e valores medidos.

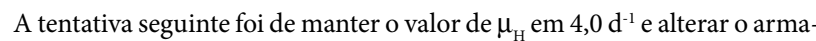
zenamento aeróbio de $\mathrm{S}_{\mathrm{S}}\left(\mathrm{K}_{\mathrm{STO}}\right)$ de $5,0 \mathrm{mg} \mathrm{DQO} \cdot \mathrm{d}^{-1} \cdot \mathrm{mg} \mathrm{DQO}^{-1}$ (valor padrão) para $7,0 \mathrm{mg} \mathrm{DQO} \cdot \mathrm{d}^{-1} \cdot \mathrm{mg} \mathrm{DQO}^{-1}$. No entanto, como não foram observadas alterações significativas, realizou-se nova simulação decrescendo o valor de $\mathrm{K}_{\text {Sто }}$ para 3,0 mg DQO $\cdot \mathrm{d}^{-1} \cdot \mathrm{mg} \mathrm{DQO}^{-1}$. Nessa simulação, pôde-se observar um aumento da fração $S_{S}$ da DQO, o que é um efeito indesejado no reator e na simulação. Sendo assim, decidiu-se por manter o valor de $\mathrm{K}_{\mathrm{STO}}$ no valor padrão e partir para a modificação de outros parâmetros.

Entretanto, mesmo com a modificação de diversos outros parâmetros, um por vez, não foi possível gerar uma simulação com curvas compatíveis estatisticamente com a curva dos dados de monitoramento do efluente do reator. Sendo assim, fez-se a tentativa de iniciar a calibração baseando-se nos valores propostos por Koch et al. (2000). Nesse, o valor padrão de $\mu_{\mathrm{H}}$ foi de $3,0 \mathrm{~d}^{-1}$. Várias tentativas foram realizadas, tanto diminuindo quanto elevando o valor de $\mu_{\mathrm{H}}$, mas não apresentaram os cenários esperados. Também foram realizadas tentativas de elevar e diminuir, um de cada vez, os valores de $\mu_{\text {Hanóx, }} \mathrm{K}_{\text {STO }}$ e a taxa de crescimento de organismos autotróficos $\left(\mu_{\mathrm{A}}\right)$. Entretanto, em nenhuma das tentativas o resultado foi de curvas estatisticamente iguais aos dados do reator em estudo, tanto para a DQO quanto para as variáveis nitrogenadas e alcalinidade.

É importante relembrar que na simulação computacional com o software ASIM foram utilizados valores de cada fração da DQO para realizar as simulações e que, como apenas análises da DQO total foram realizadas no trabalho de Morais (2015), foi necessário usar valores da literatura para estipular cada fração (Tabela 3). Sendo assim, para efeito de comparação com os valores das concentrações presentes no efluente coletado na saída do reator, as curvas simuladas de cada fração da DQO foram somadas para que se chegasse a uma estimativa da DQO total simulada. Isso dificultou a modelagem da DQO total, visto que quando se tem o conhecimento do comportamento da curva de cada fração da DQO, torna-se mais visível qual parâmetro precisa ser alterado para a calibração. E assim, calibrando-se individualmente cada fração, a soma das curvas para obtenção do comportamento da DQO total surge corretamente. Porém, não se pode afirmar que tenha sido esse o único motivo para a não validação do modelo.

Ainda, ficou claro que em todas as tentativas de calibração, as curvas de $\mathrm{X}_{\mathrm{SST}}$ jamais chegaram próximas à curva dos dados de monitoramento do sistema, o que pode ser justificado por dois fatores:

- O segundo compartimento do reator estudado é um MBBR, o que não é levado em conta pelo modelo, que o considera apenas como um reator de lodos ativados;

- A falta de inoculação inicial do reator. Portanto, era de se esperar que o reator estivesse retendo mais sólidos do que o previsto no modelo. Esses, provavelmente, ficavam aderidos ao meio suporte móvel presente no reator formando a biomassa ativa, o que foi acentuado pela falta de inoculação prévia.

Algumas vezes, mesmo após os ajustes de calibração necessários, não é possível adequar o modelo e realizar previsões de comportamento da remoção de matéria orgânica em alguns tipos de reatores como o reator em batelada sequencial (RBS) (KRISHNA; VAN LOOSDRECHT, 1999) e o MBBR. Já foram desenvolvidas várias propostas de extensões do ASM3 (MOUSSA et al., 2005; IACOPOZZI et al., 2007; KAELIN et al., 2009; ZHOU et al., 2013), no intuito de melhor caracterizar o processo de nitrificação e desnitrificação, descrevendo-os como um processo em dois estágios, ou mesmo prevendo com mais detalhes o 
crescimento e o armazenamento da biomassa. Essas extensões tornam o ASM3 mais complexo do que o modelo original. No entanto, adequando-se o modelo a cada sistema em questão, possibilitam-se melhor caracterização e controle dos processos em estações de tratamento.

Barry et al. (2017) criaram um modelo para reatores MBBR que foi validado para algumas previsões, mas que mostrou a necessidade de ajustes para as equações que descrevem o processo de fixação da DQO particulada ao biofilme. Gao, Nan e Zhang (2017) realizaram modificações no modelo ASM3 para adequá-lo a um sistema cíclico de lodos ativados (CAS) utilizando duas principais extensões: o processo simultâneo de armazenamento e crescimento e a biocinética de polímeros microbianos. Após a calibração do modelo modificado, denominado M-ASM3, houve sua validação e concluiu-se que ele se tornou mais preciso do que o ASM3 original. Neste trabalho, ficou clara a dificuldade na modelagem de reatores com biofilme, dificuldade essa que foi acrescida pela configuração compartimentada com diferentes processos em cada seção.

Wu et al. (2016) fizeram adaptações ao modelo ASM3 para utilizá-lo em uma estação de tratamento de efluente de indústria de refrigerante. Entre outros parâmetros modificados, após a calibração encontraram-se os valores de $\mu_{\mathrm{H}}$ igual a 14 $\mathrm{d}^{-1}$ e $\mu_{\mathrm{A}}$ entre 0,87 e $0,99 \mathrm{~d}^{-1}$. Koch et al. (2000) realizaram experimentos usando esgoto municipal diluído, em batelada e em reatores de lodos ativados em escala real, para calibrar e validar o modelo ASM3 nessas condições. Entre outras calibrações para as variáveis de processo, encontraram os valores de 3,0 $\mathrm{d}^{-1}$ para $\mu_{\mathrm{H}} \mathrm{e}$ de 0,9 a 2,0 $\mathrm{d}^{-1}$ para $\mu_{\mathrm{A}^{*}}$. Oselame, Fernandes e Costa (2014) utilizaram os modelos ASM1 e ASM3 para a modelagem de RBS em escala real em dois tipos de ciclos operacionais: um com apenas uma entrada de esgoto (enchimento convencional) e outro com três entradas de esgoto (enchimento escalonado). Por meio da simulação com o software ASIM, concluíram que o modelo ASM1 apresentou os melhores resultados para o ciclo com enchimento convencional. No entanto, no ciclo com enchimento escalonado, o modelo ASM3 gerou resultados mais precisos.

Notou-se que a falta de dados reais sobre os valores de cada fração da DQO do esgoto afluente ao reator aumentou a dificuldade de modelagem, visto que, mesmo utilizando-se dados da literatura de um esgoto com características similares, pequenas modificações no valor dessas variáveis alteravam consideravelmente os resultados das simulações. Neste trabalho, não foi possível a obtenção desses dados, uma vez que o reator estudado já estava fora de operação. No entanto, essa informação é de grande valia para futuras modelagens desse sistema ou de outros com configuração parecida.

Na literatura, não foram encontrados trabalhos de autores que tivessem utilizado o modelo ASM3 sem extensões para modelar reatores com as mesmas características do sistema aqui estudado. Logo, este trabalho corrobora com a necessidade da criação de extensões ao modelo que caracterizem a existência do biofilme móvel.

\section{CONCLUSÕES}

O modelo ASM3 foi utilizado para a simulação, mas não pôde ser validado, nem utilizando os valores padrão dos parâmetros cinéticos sugeridos por Henze et al. (2000), nem os valores sugeridos por Koch et al. (2000).

Por meio de processos iterativos, foram realizadas diversas modificações nos valores dos parâmetros cinéticos para calibração do modelo ASM3 ao reator em estudo, mas com nível de probabilidade de $95 \%$, não se chegou à validação do modelo.

As maiores dificuldades para validação do ASM3 ao reator podem ser creditadas ao fato de que o modelo não prevê a existência de um compartimento tipo MBBR. Sendo assim, a forma de operação do reator não se adequa ao modelo; mesmo sendo um modelo de lodos ativados, não é capaz de descrever o funcionamento da biomassa em suspensão e retida no reator, o que altera, de fato, os resultados esperados para as simulações de todas as variáveis. Ainda, ficou clara a necessidade da caracterização de cada fração da DQO do esgoto afluente para a obtenção de resultados mais próximos à realidade.

Dessa forma, sugere-se a inserção de extensões ao modelo ASM3 que prevejam e assumam o comportamento da biomassa no compartimento MBBR, bem como analisar e obter dados de cada fração da DQO afluente, para que o modelo ASM3 possa ser validado para reatores compartimentados com biomassa aderida e em suspensão, como o desta pesquisa.

\section{AGRADECIMENTOS}

Os autores agradecem à Companhia de Saneamento de Pernambuco (COMPESA) e à BRK Ambiental o suporte aos trabalhos experimentais e à coleta de amostras nas estações de tratamento de esgotos da Mangueira Recife; à Fibra Revestimentos a cessão e o suporte na operação de reatores piloto; a Luiz Galdino a ajuda na parte gráfica das figuras.

\section{CONTRIBUIÇÕES DOS AUTORES}

Peres, J.: Curadoria de Dados, Análise Formal, Investigação, Metodologia, Software, Escrita - Primeira Redação. Morais, J.: Conceituação, Curadoria de Dados, Análise Formal, Investigação, Metodologia, Administração do Projeto, Software, Validação, Visualização, Escrita - Primeira Redação. Silva Júnior, W. R.: Conceituação, Curadoria de Dados, Investigação, Software, Escrita - Primeira Redação, Escrita - Revisão e Edição. Gavazza, S.: Conceituação, Obtenção de Financiamento, Investigação, Recursos. Florencio, L.: Obtenção de Financiamento, Administração do Projeto, Recursos, Supervisão, Validação, Visualização. Kato, M. T.: Conceituação, Análise Formal, Obtenção de Financiamento, Investigação, Metodologia, Administração do Projeto, Recursos, Supervisão, Validação, Visualização, Escrita - Revisão e Edição.

\section{REFERÊNCIAS}

ACTIVATED SLUDGE SIMULATION PROGRAM (ASIM). Disponível em: https:// www.eawag.ch/en/department/eng/software/. Acesso em: 3 jan. 2016.

BARRY, U.; CHOUBERT, J:; CANLER, J.; PÉTRIMAUX, O.; HÉDUIT, A.; LESSARD, $P$. A one dimensional moving bed biofilm reactor model for nitrification of municipal wastewaters. Bioprocess Biosystem Engineering, v. 40, p. 11411149, 2017. https://doi.org/10.1007/s00449-017-1775-1
BROCKMANN, D.; BOLTZ, J.P.; MORGENROTH, E.; DAIGGER, G.T.; HENZE, M.; RITTMANN, B.; SORENSEN, K.H.; TAKACS, I.; VANROLLENGHEM, P.A.; VAN LOOSDRECHT, M. Applying a framework for calibrating a biofilm-reactor model: a full-scale moving-bed biofilm reactor active in nitrification. In: INTERNATIONAL CONFERENCE ON BIOFILM REACTORS, 9., 2013. Anais [...]. França: IWA Publishing LU, 2013. 
CHAN, Y.J.; CHONG, M.F.; LAW, C.L.; HASSEL, D.G. A review on anaerobicaerobic treatment of industrial and municipal wastewater. Chemical Engineering Journal, v. 155, n. 1-2, p. 1-18, 2009. https://doi.org/10.1016/j. cej.2009.06.041

CHRISTOFOLETTI, A. Modelagem de sistemas ambientais. São Paulo: Edgard Blucher, 2004.

FERRAI, M.; GUGLIELMI, G.; ANDREOTTOLA, G. Modelling respirometric tests for the assessment of kinetic and stoichiometric parameters on MBBR biofilm for municipal wastewater treatment. Environmental Modeling \& Software, v. 25, n. 5, p. 626-632, 2010. https://doi.org/10.1016/j. envsoft.2009.05.005

GAO, F.; NAN, J.; ZHANG, X. Simulating a cyclic activated sludge system by employing a modified ASM3 model for wastewater treatment. Bioprocess Biosystem Engineering, v. 40, n. 6, p. 877-890, 2017. https://doi.org/10.1007/ s00449-017-1752-8

GERNAEY, K.V:; VAN LOOSDRECHT, M.C.M.; HENZE, M.; LIND, M.; JØRGENSEN, S.B. Activated sludge wastewater treatment plant modelling and simulation: state of the art. Environmental Modeling \& Software, v. 19, n. 9. p. 763-783, 2004. https://doi.org/10.1016/j.envsoft.2003.03.005

GUJER, W: LARSEN, T.A. The implementation of biokinetics and conservation principles in ASIM. Water Science Technology, v. 31, n. 2, p. 257-266, 1995. https://doi.org/10.1016/0273-1223(95)00198-V

HENZE, M.; GUJER, W.; MINO, T.; VAN LOOSDRECHT, M. Activated sludge models ASM1, ASM2, ASM2d e ASM3. Londres: IWA Publishing, 2000.

IACOPOZZI, I.; INNOCENTI, V.; MARSILI-LIBELLI, S.; GIUSTI, E. A modified activated sludge model no. 3 (ASM3) with two-step nitrificationdenitrification. Environmental Modelling \& Software, v. 22, n. 6, p. 847-861, 2007. https://doi.org/10.1016/j.envsoft.2006.05.009

ISAZADEH, S.; URBINA RIVAS, L.E.; OZDURAL OZCER, P.; FRIGON, D. Reduction of waste biosolids by RAS-ozonation: model validation and sensitivity analysis for biosolids reduction and nitrification. Environmental Modelling \& Software, v. 65, p. 41-49, 2015. https://doi.org/10.1016/j.envsoft.2014.11.009

KAELIN, D:; MANSER, R.; RIEGER, L.; EUGSTER, J.; ROTTERMANN, K.; SIEGRIST, $\mathrm{H}$. Extension of ASM3 for two-step nitrification and denitrification and its calibration and validation with batch tests and pilot scale data. Water Research, v. 43, n. 6, p. 1680-1692, 2009. https://doi.org/10.1016/j.watres.2008.12.039
KOCH, G.; KÜHNI, M.; GUJER, W.; SIEGRIST, H. Calibration and validation of activated sludge model $\mathrm{n}^{03}$ for Swiss municipal wastewater. Water Research, v. 34, n. 14, p. 3580-3590, 2000.

KRISHNA, C.; VAN LOOSDRECHT, M. Substrate flux into storage and growth in relation to activated sludge modelling. Water Research, v. 33, n. 14, p. 31493161, 1999. https://doi.org/10.1016/s0043-1354(99)00031-7

MORAIS, J.C. Remoção de matéria orgânica e nitrogênio em reator compartimentado anaeróbio/anóxico e aeróbio tratando esgoto doméstico. Tese (Doutorado) - Universidade Federal de Pernambuco, Recife, 2015

MOUSSA, M.; HOOIJMANS, C.; LUBBERDING, H.; GIJZEN, H.; VAN LOOSDRECHT, M. Modelling nitrification, heterotrophic growth and predation in activated sludge. Water Research, v. 39, n. 20, p. 5080-5098, 2005. https://doi.org/10.1016/j.watres.2005.09.038

OSELAME, M.C.; FERNANDES, H.; COSTA, R.H.R. Simulation and calibration of a full-scale sequencing batch reactor for wastewater treatment. Brazilian Journal of Chemical Engineering, v. 31, n. 3, p. 649-658, 2014. https://doi. org/10.1590/0104-6632.20140313s00002541

SILVA, J.A.; SILVA, G.H.R.; SARTI, A. Tratamento de esgoto sanitário de baixa carga utilizando reator compartimentado anaeróbio/aeróbio em escala piloto. Engenharia Sanitária e Ambiental, v. 22, n. 3, p. 539-549, 2017. https:// doi.org/10.1590/s1413-41522016144668

SIN, G.; GUISASOLA, A.; PAUW, D.JW.; BAEZA, J.A.; CARRERA, J.; VANROLLEGHEM, P.A. A new approach for modelling simultaneous storage and growth processes for activated sludge systems under aerobic conditions. Biotechnology \& Bioengineering, v. 92, n. 5, p. 600-613, 2005. https://doi.org/10.1002/bit.20741

SOKAL, R.R.; ROHLF, F.J. Biometry: The Principles and Practice of Statistics in Biological Research. Journal of the Royal Statistical Society, v. 133, n. 1, p 102, 1970. https://doi.org/10.2307/2343822

WU, X.; YANG, Y.; WU, G.; MAO, J.; ZHOU, T. Simulation and optimization of a coking wastewater biological treatment process by activated sludge models (ASM). Journal of Environmental Management, v. 165, p. 235-242. 2016. https://doi.org/10.1016/j.jenvman.2015.09.041

ZHOU, M.; GONG, J.; YANG, C.; PU, W. Simulation of the performance of aerobic granular sludge SBR using modified ASM3 model. Bioresource Technology, v. 127, p. 473-481, 2013. https://doi.org/10.1016/j.biortech.2012.09.076 